\title{
An Analytical Method to Calculate Phantom Scatter Factor for Photon Beam Accelerators
}

\author{
Mohammad Javad Tahmasebi Birgani ${ }^{1}$, Nahid Chegeni ${ }^{2}$, Mohammad Ali Behrooz ${ }^{3}$, Marziyeh Bagheri ${ }^{4}$, Amir \\ Danyaei $^{2}$, Azin Shamsi ${ }^{5}$
}

${ }^{1}$ Ph.D. of Medical Physics, Professor, Department of Medical Physics and Radiation Therapy and Medical Physics Department, Golestan Hospital, Jundishapur University of Medical Sciences, Faculty of Medicine, Ahvaz Jundishapur University of Medical Sciences: Ahvaz, Iran

${ }^{2}$ Ph.D. of Medical Physics, Assistant Professor, Department of Medical Physics, Faculty of Medicine, Ahvaz Jundishapur University of Medical Sciences: Ahvaz, Iran

${ }^{3}$ Ph.D. of Bio Physics, Professor, Department of Medical Physics, Faculty of Medicine, Ahvaz Jundishapur University of Medical Sciences: Ahvaz, Iran

${ }^{4}$ M.Sc. of Medical Physics, Department of Medical Physics, Faculty of Medicine, Ahvaz Jundishapur University of Medical Sciences: Ahvaz, Iran

${ }^{5}$ M.Sc. of Medical Physics, Radiation Therapy and Medical Physics Department, Golestan Hospital, Jundishapur University of Medical Sciences

\section{Type of article: Original}

\begin{abstract}
Introduction: One of the important input factors in the commissioning of the radiotherapy treatment planning systems is the phantom scatter factor (Sp) which requires the same collimator opening for all radiation fields. In this study, we have proposed an analytical method to overcome this issue.

Methods: The measurements were performed using Siemens Primus Plus with photon energy 6 MV for field sizes from $5 \times 5 \mathrm{~cm}^{2}$ to $40 \times 40 \mathrm{~cm}^{2}$. Phantom scatter factor was measured through the division of total scatter output factors (Scp), and collimator scatter factor (Sc).

Results: The mean percent difference between the measured and calculated $\mathrm{Sp}$ was $1.00 \%$ and $-3.11 \%$ for $5 \times 5$, $40 \times 40 \mathrm{~cm}^{2}$ field size respectively.

Conclusion: This method is applicable especially for small fields used in IMRT which, measuring collimator scatter factor is not reliable due to the lateral electron disequilibrium.

Keywords: Radiotherapy, Total scatter factor, Phantom scatter factor
\end{abstract}

\section{Introduction}

In radiation therapy, the calculation of the monitor unit (MU) is necessary to deliver a prescribed absorbed dose for the planning target volume (PTV) (1). One of the important factors to determine MU is the scatter factor. According to Kahn et al. the collimator scatter factor $(\mathrm{Sc})$ is commonly called the output factor or head scatter factor, and may be defined as the ratio of the output in air for a given field to that for the reference field (e.g., $10 \times 10 \mathrm{~cm}^{2}$ ) with a buildup cap of a size large enough to provide electronic equilibrium (2). To prevent contaminating electrons to the detector, the columnar mini phantom is used instead of buildup cap (3). The Sp is defined as the ratio of the absorbed dose at the depth of maximum dose (dmax) for a given field to that in the reference field at the same depth with the same collimator opening (4). The Sp becomes difficult to measure in practice (5); An indirect method of determining $\mathrm{Sp}$, relates the total scatter factor (Scp) to the collimator scatter factor (Sc) (Figure 1, Eq.1); where Scp (r) is defined as the ratio of the absorbed dose at dmax in a water phantom for specific field to the absorbed dose in a reference field (6-10). Most treatment planning systems (TPS) need to enter Scp, Sc and Sp. Error in the measurement of Scp and Sc will cause problems to drive $\mathrm{Sp}$ (11).Therefore, it is necessary to calculate directly the phantom scatter factor using

\section{Corresponding author:}

Assistant Professor Dr. Nahid Chegeni, Department of Medical Physics, Faculty of Medicine, Ahvaz Jundishapur University of Medical Sciences: Ahvaz, Iran. Tel: +98.916 353 4022, Email: chegenin@gmail.com

Received: September 06, 2016, Accepted: January 03, 2017, Published: January 2017

iThenticate screening: November 30, 2016, English editing: January 06, 2017, Quality control: January 10, 2017

(C) 2017 The Authors. This is an open access article under the terms of the Creative Commons Attribution-NonCommercialNoDerivs License, which permits use and distribution in any medium, provided the original work is properly cited, the use is noncommercial and no modifications or adaptations are made. 
Monte Carlo simulation or analytical methods. According to the Sp definition, each measurement in the phantom has scattering effects from both phantom and collimator together. To eliminate the effect of collimator in this measurement, the field collimator should be tried to be constant during the measurement process. To solve this issue, Khan et al. produced the treatment field in phantom by collimator and then, without a change in collimator, they created the field references by lead blocks (12); Then, for the same condition, they defined the ratio of dosimeter readings for these two fields as Sp regardless of the block scattering involved in the measurement. An analytical model for calculating $\mathrm{Sp}$ is presented in this paper. We proposed a new setting to achieve the same collimator opening and all measuring done in phantom, therefore the lack of the lateral electron equilibrium for small field is not an issue.

(1) $S p \approx \frac{S c p}{S c}$

(2) $\operatorname{Sc}(L)=\frac{\operatorname{cop} R\left(c_{L}, 100, L\right) / M}{\operatorname{cop} R\left(c_{0}, 100, L_{0}\right) / M}$

(3) $\operatorname{Scp}(L)=\frac{d_{\max } R\left(c_{L}, 100, L\right) / M}{d_{\max } R\left(c_{0}, 100, L_{0}\right) / M}$

(7) $\alpha=$
(4) $\operatorname{Scp}(L)=\frac{{ }_{d} R\left(c_{L}, 100, L\right)}{{ }_{d} R\left(c_{0}, 100, L_{0}\right)} \times \frac{P D D\left(100, L_{0}, d\right)}{P D D(100, L, d)}$

(5) $\operatorname{Sp}(L)=\frac{d_{m} R\left(c_{0}, 100, L\right)}{{ }_{d_{m}} R\left(c_{0}, 100, L_{0}\right)}$

(6) $S_{p}(L)=\alpha S_{c p}(L)$

(8) i) $\mu=0$ (without attenuation) ii) $\mu(\mathrm{r})=0.0473\left(1+0.00033 \mathrm{r}^{2}\right.$ ) (which $\mathrm{r}$ is the radius of the field) (17) iii) $\mu(d, L)=\frac{A_{L} B_{L} e^{-B_{L} \cdot d}-C_{L} D_{L} e^{-D_{L} \cdot d}}{A_{L} e^{-B_{L} \cdot d}-C_{L} e^{-D_{L} \cdot d}}$ (which $\mathrm{d}, \mathrm{L}$ are depth and field size respectively and $\mathrm{A}_{\mathrm{L}}, \mathrm{B}_{\mathrm{L}}, \ldots$ are functions of L) (18)

$$
\int_{-Y}^{Y} \int_{-X}^{X} \frac{e^{-\mu \sqrt{x^{2}+y^{2}}} d x d y}{x^{2}+y^{2}}=
$$

(9)

$$
4\left[\iint_{\text {region } 1} \frac{e^{-\mu r} r d r d \theta}{r^{2}}+\iint_{\text {region } 2} \frac{e^{-\mu r} r d r \cdot d \theta}{r^{2}}\right]=4\left[\int_{\theta=0}^{\tan ^{-1} \frac{Y}{X}} \int_{\varepsilon \rightarrow 0}^{\frac{X}{\cos \theta}} \frac{e^{-\mu r} d r d \theta}{r}+\int_{\tan ^{-1} \frac{Y}{X}}^{\frac{\pi}{2}} \int_{\varepsilon \rightarrow 0}^{\frac{Y}{\sin \theta}} \frac{e^{-\mu r} d r d \theta}{r}\right]
$$

Figure 1. Formula in the study

\section{Material and Methods}

\subsection{Theory}

According to the definition, the in-air output factor $(\mathrm{Sc})$ can be written as Figure 1, Eq.2; which numerator and denominator are chamber readings $(\mathrm{R})$ for the same number of monitor units $(\mathrm{M})$ in the presence of a build-up cap for a field with dimensions $\mathrm{L}_{\text {and }} \mathrm{L}_{0}$ (as a reference field e.g. $10 \times 10 \mathrm{~cm}^{2}$ ) at $\mathrm{SSD}=100 \mathrm{~cm}$ and collimator opening $\mathrm{C}_{\mathrm{L}}, \mathrm{C}_{0}$ respectively. For the total scatter factor, measuring at the $\mathrm{d}_{\max }$ in water phantom for a specific field (L) to that reference field $\left(\mathrm{L}_{0}\right)$ for the same number of monitor units $(\mathrm{M})(13)$, it would be written as Figure 1, Eq.3. To prevent electron contamination of the photon beam, it is recommended that all readings are performed at a depth of $\mathrm{d}=10 \mathrm{~cm}$ and then they are transmitted to the maximum depth using PDD (d) for that field size (14). So, the formula in Figure 1, Eq.4. According to Figure 2., L, $\mathrm{L}_{0}$ (field size), $\mathrm{C}_{\mathrm{L}}$, and $\mathrm{C}_{0}$ (collimator opening) are shown as $\mathrm{AB}, \mathrm{EF}, A \hat{O} B$, and $E \hat{O} F$ respectively. As can be seen in Figure 2, the total scatter factor is obtained by dividing the dosimeter reading which is caused by primary and secondary radiation $\left(S^{\prime}\right)$ from two volume $V_{\mathrm{ABCD}}$ and reference volume $\mathrm{V}_{\mathrm{EFGH}}$ and then correct for depth $d$ to $d_{\max }$. The $\mathrm{Sp}$ is defined as the ratio of the absorbed dose at the $\mathrm{d}_{\max }$ in phantom (water or solid water) 
for a given field to that in the reference field with the same collimator opening $\left(\mathrm{C}_{0}\right)$ to eliminate the collimator effect (4). Therefore, the formula in Figure 1, Eq.5. In this study, to maintain the collimator opening for all fields, the phantom surface can be pulled up (for $\mathrm{L}<\mathrm{L} 0$ ) or down (for $\mathrm{L}>\mathrm{L} 0$ ) (Figure 2). For example, for a field smaller than $\mathrm{L}_{0}$, $\mathrm{L}=\mathrm{AB}$, it is necessary to pull up phantom surface to $\mathrm{J}=(\mathrm{L} / \mathrm{L} 0) \times \mathrm{SSD}$ to achieve the same collimator opening. However, care must be which the dosimeter reading at $\mathrm{J}^{\prime}$ needs to be corrected for SSD changes using Mayneord Factor. Therefore; we have to perform dosimetry in several SSDs and the main problem is phantom surface collision with Linac head for small fields. On the other hand, according to Eq. 3 and 5, the difference between the Sp and the total scatter factor is the amount of the collimator opening. Therefore, by considering a correction factor (for the effect of collimator) in the total scatter factor (Eq. 4), Sp can be achieved (Eq.5) as Figure 1, Eq.6. In Figure 2 with regards to the Pythagorean theorem, the trapezoid $A B C{ }^{\prime \prime} D^{\prime \prime}$ and $A^{\prime} B^{\prime} C^{\prime} D^{\prime}$ are equal; therefore, the $S p$ is obtained by dividing the

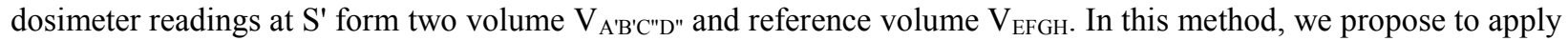
a factor $(\alpha)$ to correct scatter portion reached to the dosimeter $\left(S^{\prime}\right)$ from $V_{A B C D}$ to $V_{A B C} D^{\prime}$. As mentioned in previous studies $(15,16)$, suppose that there is a parallel beam striking the surface of the phantom and consider a reference plane normal to the central axis, placed at depth $\mathrm{d}=10 \mathrm{~cm}$ which the origin is on the central axis. Hence, each element (dx.dy) on this plane acts as a source of scattered radiation. The amount of scatter radiation reaching the central axis (dosimeter location, $S^{\prime}$ ) is inversely proportional to the square of the distance between surface elements and the origin. Therefore, with taking into account the photon attenuation $\mu, \alpha$ is defined as Figure 1, Eq.7; Where $\mu$ is energy attenuation coefficient which depends on energy, field size and depth $(17,18)$. Therefore, the formula in Figure 1, Eq.8. $\alpha$ will be greater or lesser than 1 while phantom surface was pulled up or down respectively. Let us eliminate the small area at the origin of the coordinate with radius $\varepsilon$ (refers to the ion chamber radius $=3 \mathrm{~mm}$ ) to overcome singularity in nominator and denominator in Eq.7 and use polar system (Figure 3). Therefore, the scatter contribution to dosimeter location $\left(\mathrm{S}^{\prime}\right)$ for a rectangular field with dimensions $(2 \mathrm{Y} \times 2 \mathrm{X})$ (Figure 3) is calculated as Figure 1, Eq.9. Finally, a general computer program using MATLAB 7.12.0 software was written to calculate $\alpha$ using the numerical method to solve Eq. 9.

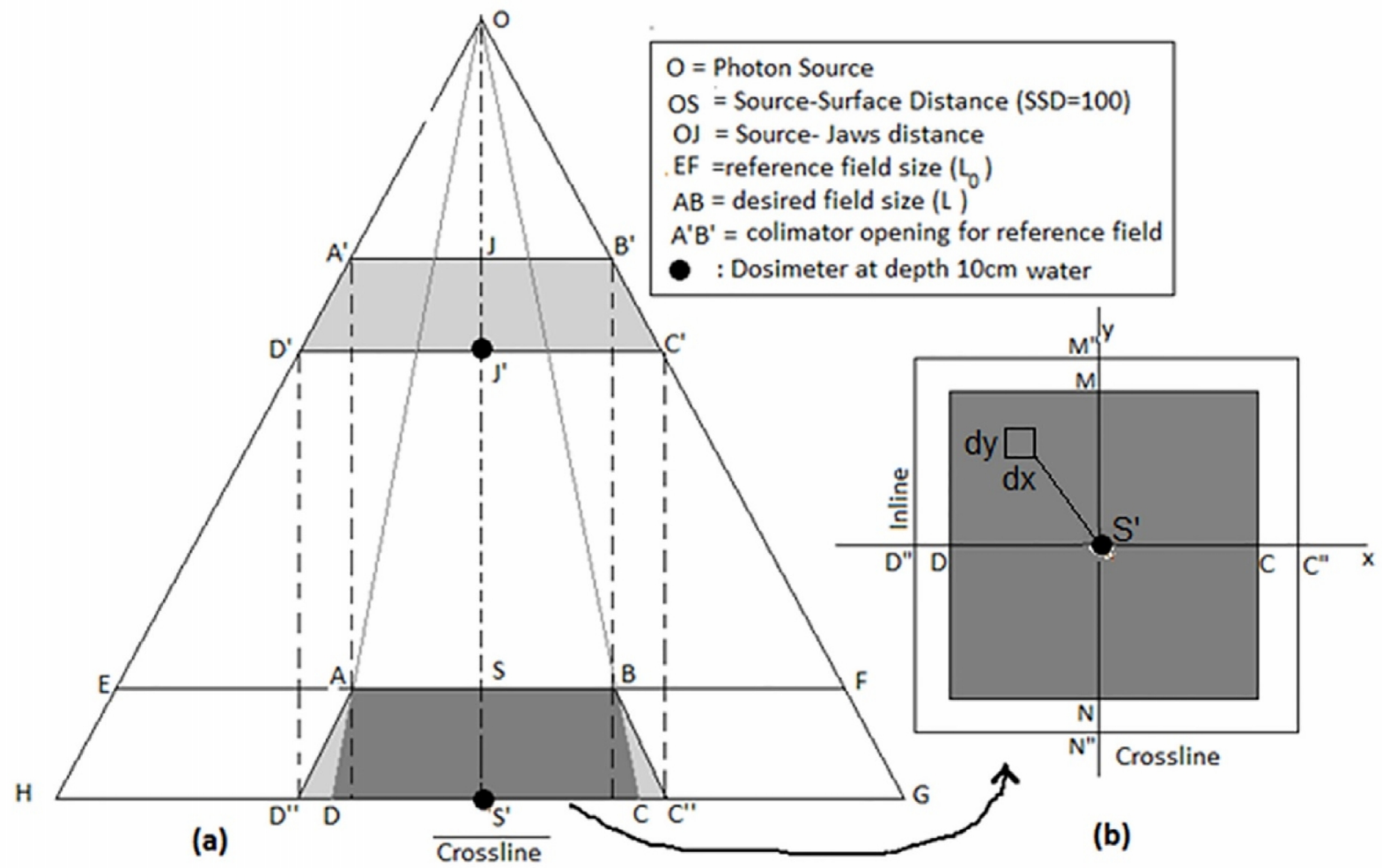

Figure 2. (a) Diagram of under-radiation treatment volume in the standard mode of $S S D=100$ for reference field $\left(\mathrm{V}_{\mathrm{EFGH}}\right)$ and a small field $\left(\mathrm{V}_{\mathrm{ABCD}}\right)$. In order to maintain collimator opening for smaller fields than the reference field, the phantom surface can be pulled up to the collimator location (A'B'). (b) DMCN, D"M"C"N" are the projection the field size $\mathrm{L}$ at distance $\mathrm{OS}$ ', OJ'. 


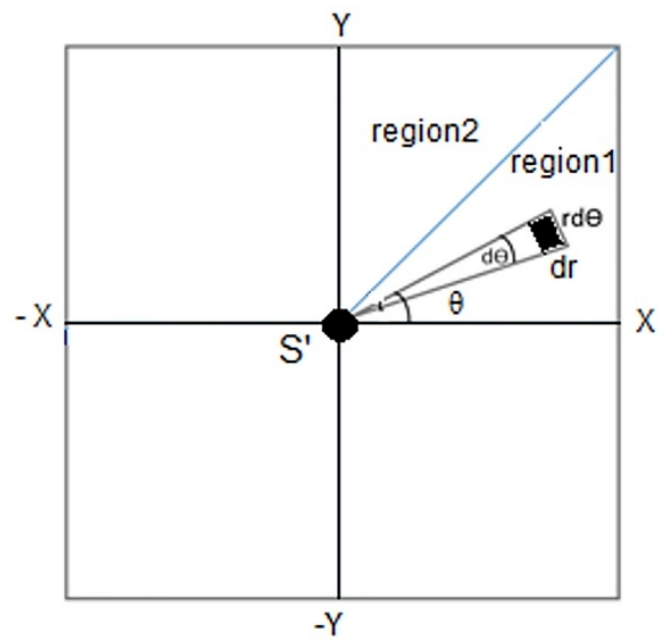

Figure 3. Polar system to calculate the scatter contribution to dosimeter location ( $\left.\mathrm{S}^{\prime}\right)$ for a rectangular field with dimensions $(2 \mathrm{Y} \times 2 \mathrm{X})$.

\subsection{Dosimetry and measurements}

The linear accelerator used in this study was a Siemens Primus Plus with photon energy 6 MV. A Scanditronix blue phantom (Wellhofer, Germany) $(50 \mathrm{~cm} \times 50 \mathrm{~cm} \times 50 \mathrm{~cm})$ and the Farmer ionization chamber $0.6 \mathrm{cc}$ were applied for measuring. Total scatter output factors, Scp, were measured at 10-cm depth water ( $S$ ' in Figure 2) and source- surface distance (SSD) $100 \mathrm{~cm}$ and for field sizes from $5 \times 5 \mathrm{~cm}^{2}$ to $40 \times 40 \mathrm{~cm}^{2}$. For Sc measurements, a polystyrene build-up cap was employed at maximum depth and source-surface distance (SSD) 100-cm. The Sp was measured indirectly using Eq. 1 as a routine method and then compared with Sp which was calculated by Eq.6, 7.

\section{Results}

As has been shown in Table 1, the Sp for the photon beam was indirectly measured for all field sizes using measuring Scp and Sc according to Eq.1. The correction factor in Eq. $6(\alpha)$ was calculated using numerical calculation then by multiplying to Scp, Sp-cal was obtained. As can be seen, variations regarded to the energy attenuation coefficient $(\mu)$ changes Sp-cal results. While, elimination of the photon attenuation shows better results (fourth column). The measured and calculated phantom scatter were compared by percentage of difference which is mentioned the last three columns. According to Figure 4. for fields, which are far from the reference field (e. g. 5 and $40 \mathrm{~cm}$ ), the differences between calculation and measurement are further than fields next to the reference field. This difference for fields larger than the reference field is becoming more evident.

Table 1. Phantom scatter factor (Sp) measured and calculated based on routine method (Sp-meas $=\mathrm{Scp} / \mathrm{Sc})$ and analytical method $(\mathrm{Sp}-\mathrm{cal}=\alpha . \mathrm{Scp})$.

\begin{tabular}{|c|c|c|c|c|c|c|c|c|c|}
\hline \multirow{2}{*}{$\begin{array}{l}\mathrm{L}(\mathrm{cm}) \\
\text { (Field size) }\end{array}$} & \multirow[t]{2}{*}{$\mathrm{Sc}$} & \multirow[t]{2}{*}{ Scp } & \multirow[t]{2}{*}{ Sp-meas $(\mathrm{Scp} / \mathrm{Sc})$} & \multicolumn{3}{|c|}{ Sp-cal $=\alpha . S c p$} & \multicolumn{3}{|c|}{ Diff $\%\left(\mathrm{Sp}_{\text {meas }}-\mathrm{Sp}_{\text {cal }}\right) / \mathrm{Sp}_{\text {meas }}$} \\
\hline & & & & $\mu=0$ & $\mu(\mathrm{r})^{+}$ & $\mu(d, L)^{*}$ & $\mu=0$ & $\mu(\mathrm{r})^{+}$ & $\mu(d, L)^{*}$ \\
\hline $5 \times 5$ & 0.957 & 0.948 & 0.990 & 0.983 & 0.980 & 0.978 & 0.72 & 1.05 & 1.23 \\
\hline $6 \times 6$ & 0.971 & 0.963 & 0.992 & 0.986 & 0.983 & 0.982 & 0.62 & 0.87 & 1.01 \\
\hline $7 \times 7$ & 0.982 & 0.976 & 0.994 & 0.990 & 0.988 & 0.987 & 0.39 & 0.57 & 0.67 \\
\hline $8 \times 8$ & 0.991 & 0.993 & 1.002 & 1.001 & 1.000 & 0.999 & 0.10 & 0.22 & 0.28 \\
\hline $9 \times 9$ & 0.998 & 0.999 & 1.001 & 1.002 & 1.002 & 1.002 & -0.14 & -0.09 & -0.06 \\
\hline $10 \times 10$ & 1.000 & 1.000 & 1.000 & 1.000 & 1.000 & 1.000 & 0.00 & 0.00 & 0.00 \\
\hline $12 \times 12$ & 1.011 & 1.018 & 1.007 & 1.013 & 1.014 & 1.015 & -0.62 & -0.72 & -0.77 \\
\hline $15 \times 15$ & 1.021 & 1.038 & 1.017 & 1.029 & 1.031 & 1.032 & -1.18 & -1.43 & -1.54 \\
\hline $20 \times 20$ & 1.029 & 1.058 & 1.028 & 1.045 & 1.049 & 1.051 & -1.61 & -2.07 & -2.24 \\
\hline $25 \times 25$ & 1.035 & 1.081 & 1.044 & 1.066 & 1.072 & 1.074 & -2.02 & -2.67 & -2.87 \\
\hline $30 \times 30$ & 1.037 & 1.089 & 1.050 & 1.072 & 1.081 & 1.083 & -2.13 & -2.94 & -3.14 \\
\hline $35 \times 30$ & 1.040 & 1.091 & 1.049 & 1.074 & 1.084 & 1.086 & -2.37 & -3.32 & -3.51 \\
\hline $40 \times 40$ & 1.040 & 1.104 & 1.062 & 1.086 & 1.098 & 1.100 & -2.33 & -3.42 & -3.59 \\
\hline
\end{tabular}

$+\mu$ has been extracted from Bjangard's study; $* \mu$ has been extracted from Tahmasebi's study 


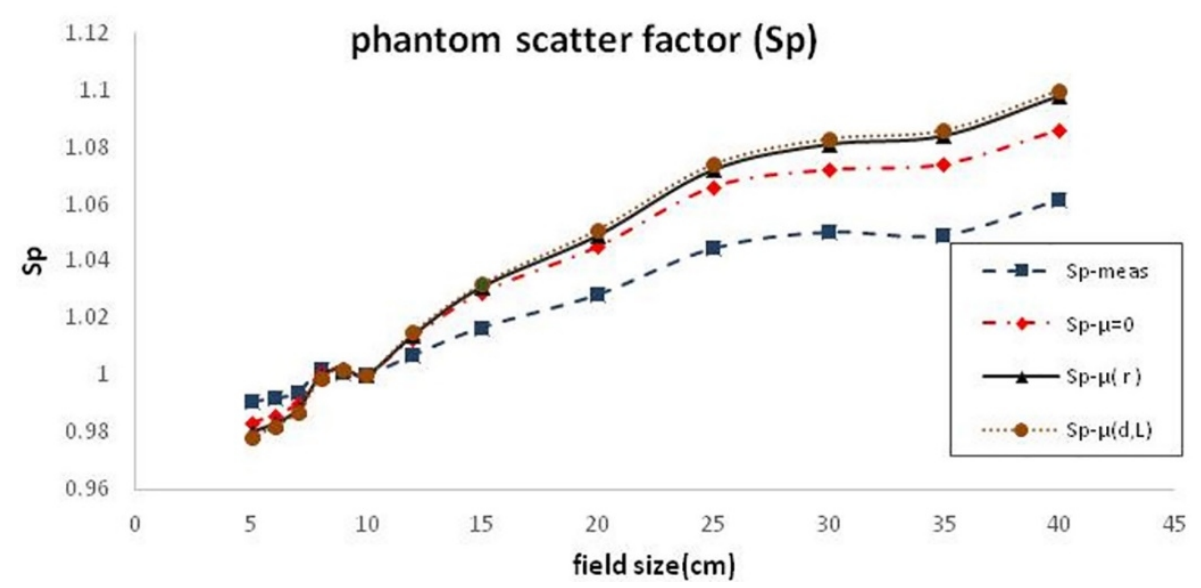

Figure 4. Phantom scatter factor $(\mathrm{Sp})$ measured and calculated using various energy attenuation formula.

\section{Discussion}

The phantom scatter correction factor $(\mathrm{Sp})$ accounts for the contribution of the radiation scattered in the phantom material to the dose at the point at depth $\mathrm{d}_{\mathrm{ref}}$ on the central axis that in the reference irradiation set-up. The amount of scattered radiation reaching to the dosimeter ( $\left.\mathrm{S}^{\prime}\right)$ depends on the volume of the phantom irradiated material, therefore, $\mathrm{Sp}$ is as a function of the field size at the surface of the phantom. There is little literature which has discussed analytical expression to calculate Sp. Blais and Karabrahimi in 2006 applied an equivalent field and Clarkson's method to calculate collimator scatter factor and Sp for kilovoltage $\mathrm{x}$-ray radiation fields. They reported that the percentage difference between calculated and measured $\mathrm{Sp}$ was $6.3 \%$ and 3.5\% for the equivalent field and Clarkson's method respectively(19). Chung et al. provided an analytical formalism to calculate phantom scatter factors for flattening filter free (FFF) mode photon beam by taking the product of the $\mathrm{Sp}$ in the presence of the flattening filter (FF) and the ratio of the off-axis ratio between the FFF mode and FF mode. They concluded that the percent difference between calculated and measured Sp ranged from $-0.34 \%$ to $0.73 \%$ for Varian $6 \mathrm{MV}(20)$. This study has attempted to present an analytical approach to calculate the Sp using Scp values. It is most important to consider the percent difference range between the indirect measured and calculated Sp with $0.72 \%, 1.05 \%$ and $1.23 \%$ for $5 \times 5 \mathrm{~cm}^{2}$ field size and $2.33 \%,-3.42 \%$ and $-3.59 \%$ for $40 \times 40 \mathrm{~cm}^{2}$ field size for $\mu=0, \mu(\mathrm{r})$, and $\mu(\mathrm{d}, \mathrm{L})$ respectively. As can be seen in the last two columns Table.1, the Sp calculation shows approximately the same results for two expressions for $\mu$. McKerracher showed indirect $\mathrm{Sp}$ values for fields $>4 \mathrm{~cm}$ width are independent of beam defining system and dependent only on measurement depth, beam quality and beam area irradiated, and $\mathrm{Sp}$ in fields $<4 \mathrm{~cm}$ width has also been shown to be independent of beam defining system and Linac design, and dependent only on measurement depth and beam area irradiated (6). One of the problems of indirect measurement method is measuring Sc for small fields due to the lack of the electron equilibrium. For small fields, this method is also insufficient due to the correction factor $(\alpha)$ will be close to 1 , therefore, some other correction factors most be considered.

\section{Acknowledgments:}

This study is taken from the Master's thesis therefore, the authors thank the office of Vice-Chancellor for Research of Jundishapur University of Medical Science, Ahvaz, Iran, for financial support with grant number (U-94039).

\section{Conflict of Interest:}

There is no conflict of interest to be declared.

\section{Authors' contributions:}

All authors contributed to this project and article equally. All authors read and approved the final manuscript.

\section{References:}

1) Miyashita H, Hatanaka S, Fujita Y, Hashimoto S, Myojyoyama A, Saitoh H. Quantitative analysis of in-air output ratio. J Radiat Res. 2013; 54(3): 553-60. doi: 10.1093/jrr/rrs118. PMID: 23292148, PMCID: PMC3650743.

2) Khan FM, Gibbons JP. Khan's the physics of radiation therapy: Lippincott Williams \& Wilkins; 2014. 
3) Zhu TC, Ahnesjö A, Lam KL, Li XA, Ma CM, Palta JR, et al. Report of AAPM Therapy Physics Committee Task Group 74: in-air output ratio, Sc, for megavoltage photon beams. Med Phys. 2009; 36(11): $5261-91$. doi: 10.1118/1.3227367. PMID: 19994536.

4) Khan FM. The physics of radiation therapy. 4 ed. med Phy; 2012. 148-52.

5) Iftikhar A. Measurements of output factors using different ionization chambers and build up caps. Iranian Journal of Radiation Research. 2012; 10(2): 95-8.

6) McKerracher C, Thwaites DI. Phantom scatter factors for small MV photon fields. Radiotherapy and Oncology. 2008; 86(2): 272-5. doi: 10.1016/j.radonc.2007.10.040. PMID: 18061694.

7) Van Gasteren JJ, Heukelom S, Van Kleffens HJ, Van der Laarse R, Venselaar JL, Westermann CF. The determination of phantom and collimator scatter components of the output of megavoltage photon beams: measurement of the collimator scatter part with a beam-coaxial narrow cylindrical phantom. Radiother Oncol. 1991; 20(4): 250-7. doi: 10.1016/0167-8140(91)90124-Y. PMID: 1906190.

8) Hayakawa T, Yamada T, Sakai H, Kasahara T, Inoue T, Miyakawa M. [Estimation of the phantom scatter factor (Sp) of rectangular fields]. Nihon Hoshasen Gijutsu Gakkai zasshi. 2011; 68(1): 15-29. doi: 10.6009/jjrt.2012-JSRT-68.1.15. PMID: 22277812.

9) Oh YK, Choi TJ, Kim JH, Kim OB. Determination of Phantom Scatter Factors for Small Photon Fields. Korean J Med Phys. 2009; 20(2): 106-11.

10) Richmond N, Allen V, Daniel J, Dacey R, Walker C. A comparison of phantom scatter from flattened and flattening filter free high-energy photon beams. Med Dosim. 2015; 40(1): 58-63. doi: 10.1016/j.meddos.2014.10.001. PMID: 25454113.

11) Tailor RC, Followill DS, Hernandez N, Zhu TS. Quality-Assurance Check of Collimator and PhantomScatter Factors. Medical Physics; 2003.

12) Khan FM, Sewchand W, Lee J, Williamson JF. Revision of tissue - maximum ratio and scatter - maximum ratio concepts for cobalt 60 and higher energy $\mathrm{x}$ - ray beams. Med Phys. 1980; 7(3): 230-7. doi: 10.1118/1.594648. PMID: 6771511.

13) Venselaar JL, Heukelom S, Jager HN, Mijnheer BJ, van Gasteren JJ, van Kleffens HJ, et al. Is there a need for a revised table of equivalent square fields for the determination of phantom scatter correction factors? Phys Med Biol. 1997; 42(12): 2369-81. doi: 10.1088/0031-9155/42/12/005. PMID: 9434294.

14) Venselaar JL, Van Gasteren JJ, Heukelom S, Jager HN, Mijnheer BJ, Van der Laarse R, et al. A consistent formalism for the application of phantom and collimator scatter factors. Phys Med Biol. 1999; 44(2): 365 81. doi: 10.1088/0031-9155/44/2/006. PMID: 10070788 .

15) Tahmasebi Birgani MJ, Behrouz MA, Aliakbari S, Hosseini SM, Khezerloo D. Determination of square equivalent field for rectangular field in electron therapy. J Med Phys. 2013; 38(2): 82-6. doi: 10.4103/09716203.111317. PMID: 23776311, PMCID: PMC3683305.

16) Tahmasebi Birgani MJ, Chegeni N, Zabihzadeh M, Hamzian N. An analytical method to calculate equivalent fields to irregular symmetric and asymmetric photon fields. Med Dosim. 2014; 39(1): 54-9. doi: 10.1016/j.meddos.2013.09.007. PMID: 24485053.

17) Bjärngard BE, Shackford H. Attenuation in high - energy x - ray beams. Medical Physics. 1994; 21(7): 1069-73. doi: 10.1118/1.597349. PMID: 7968838.

18) Tahmasebi Birgani M, Behrooz MA, Shahbazian H, Shams A. Determination of the attenuation coefficient for megavoltage photons in the water phantom. Iranian journal of radiation research (IJRR). 2012; 9(4): 251 5.

19) Karabrahimi V, Blais N. SU-FF-T-366: Prediction of Collimator Scatter Factor and Phantom Scatter Factor for Kilovoltage X-Ray Radiation Fields. Medical Physics. 2006; 33(6). doi: 10.1118/1.2241286.

20) Chung H, Prado KL, Yi BY. An analytical formalism to calculate phantom scatter factors for flattening filter free (FFF) mode photon beams. Phys Med Biol. 2014; 59(4): 951-60. doi: 10.1088/0031-9155/59/4/951. PMID: 24503449. 